\title{
Posterior Stabilized Designs in Modern Total Knee Arthroplasty: Vestigial Organs
}

\author{
Michael Stefl, $\mathrm{MD}^{2}$
}

R. Michael Meneghini MD ${ }^{1,2}$

${ }^{1}$ Indiana University Health Physicians Orthopedics \& Sports Medicine, Fishers, IN

${ }^{2}$ Indiana University School of Medicine, Department of Orthopaedic Surgery, Indianapolis, IN

R. Michael Meneghini,MD (corresponding author)

Indiana University School of Medicine, Department of Orthopaedic Surgery

Indiana University Health Orthopedics, 13000 East 136th Street, Suite 2000, Fishers, IN 46037

Phone: 317-688-5980; Fax: 317-678-3222

Email: rmeneghi@iuhealth.org

This is the author's manuscript of the article published in final edited form as:

Stefl, M., \& Meneghini, R. M. (2019). Posterior Stabilized Designs in Modern Total Knee Arthroplasty: Vestigial Organs. Seminars 


\section{Abstract}

Clinical difference between posterior-stabilized (PS) and cruciate-retaining (CR) designs in total knee arthroplasty has remained elusive for decades. This classic debate has been given pause due to futile efforts to prove one design superior over the other. Recently, anterior-lipped and more conforming $\mathrm{CR}$ bearings have emerged to substitute for the posterior-cruciate ligament (PCL), if absent, damaged or resected, and obviate the need for the archaic cam-post mechanism of a traditional PS design. Advantages of avoiding a PS TKA include eliminating the risk of box cut induced femoral condylar fracture, operative efficiency by removing procedural steps, removing the articulation that is a source of wear, post deformation, breakage, or dislocation, and eliminating patellar clunk.

\section{Introduction}

The debate over superiority of posterior-stabilized (PS) and cruciate-retaining (CR) bearing articulations in total knee arthroplasty (TKA) has persisted since the inception of both designs in the 1970's. Howeyer, proponents of each design were unable to definitely claim superiority due to inadequate scientific rigor in research studies and utilization of outcome measures unable to discern subtle differences between implant designs. Originally, studies focused on survivorship rather than patient reported outcomes, and the pros and cons of these articulation bearings were largely theoretical. Recently, anterior-lipped and more conforming CR bearings have emerged to substitute for the PCL if absent, damaged or resected, and obviate the need for the archaic cam-post mechanism of a traditional PS design. This manuscript will explore the disadvantages of PS articulations as well as review the modern outcome and 
survivorship data showing equivalent or improved results with CR-type articulations compared to PS articulations.

\section{Disadvantages of Posterior Stabilized Articulations}

Posterior Stabilized articulations were designed to rely on a cam and post mechanism to produce femoral rollback, in order to attempt to duplicate the natural kinematics of the knee. This cam and post mechanism has resulted in a variety of unique complications attributable to its inherent design structure and mechanics.

Femoral condylar fracture can occur in PS TKA designs due to either the box cut itself or the stress riser created between the medial and lateral box corners and the metaphyseal cortices and can occur during the bone cuts, with insertion of implant trials, cementation of the final implant or postoperatively. Although this complication has been shown by Alden et al to be rare ( $0.39 \%$ risk of intraoperative fracture during TKA), it can be detrimental to patient recovery and outcomes[1]. CR-type designs do not necessitate a femoral box cut, which has also been shown to decrease operative time by removing this additional step in the procedure. This was shown by Scott and Smith, with statistically significantly decreased tourniquet time when comparing CRtype and PS-type articulations [2]. The cam and post mechanism is also a potential source of polyethylene wear and fatigue failure, being a mechanical articulation under stress. Breakage via fatigue failure of this mechanism has been observed (Figure 1) and reported in multiple PS bearing designs [3, 4]. Dislocation also may occur in PS design knees, usually posteriorly but occasionally anteriorly [5]. The box mechanism also may lead to a complication unique to PS designs, patellar clunk. Newer designs that incorporate a deep trochlear groove and a smooth transition of the intercondylar box have improved results by minimizing the incidence of patellar 
clunk [6], yet complete elimination remains impossible due to the inherent geometrical shape required of a cam-post mechanism.

\section{Outcome and Survivorship Results}

Over the past decade, modern more conforming CR bearings have emerged with intent of substituting for the PCL with a more pronounced anterior lip or conforming polyethylene articulation. These modern enhanced bearings with greater conformity contrast with traditional flat CR bearings and clinical data has emerged with promising results. This data shows equivalent or improved functional outcomes as compared to PS design articulations and consists of retrospective cohort studies, randomized prospective studies, large institution registry data and national registry level data.

In a retrospective cohort analysis, Biyani et al evaluated a series of 39 PS design knees compared to 43 cruciate stabilizing (anterior-lipped termed "CS") design knees that had anteriorlipped inserts [7]. The PCL was resected in all patients in the series. Despite the PCL being absent, there were no differences in any functional outcomes at minimum 1-year follow-up. This supports the hypothesis that an anterior-lipped insert is an adequate functional substitute for a post-cam articulation in patients undergoing TKA with PCL excision. This is not a new finding and was also described by Parsley et al in 2006 [8]. This group compared 121 PS design knees to 88 ultracongruent CS design, PCL sacrificed knees and found equivalence in all outcome scores at one year, again showing no evidence requiring posterior stabilization in PCL sacrificing TKAs. This was again shown by Laskin et al who randomized 176 patients into PS design TKA versus ultracongruent CS design TKA with the PCL resected and found that there was no difference in functional outcomes [9]. 
A prospective randomized trial by Sur et al evaluated patients who underwent bilateral TKA with a PS bearing in one knee and a CS bearing with PCL resected in the contralateral knee. The CS group had more posterior translation than the PS group; However, no functional outcome differences between knee designs at one-year follow-up were observed [10]. In a prospective randomized controlled trial, Scott and co-authors compared 56 PS TKAs to 55 TKAs with anterior-lipped CS inserts and found no functional outcome differences at minimum twoyear follow up [2] with tourniquet times significantly longer in the PS group [2]. Their five-year follow-up data of the same cohorts continues to show no functional outcome differences and does show decreased mechanical symptoms in the CS group, compared to the PS cam-post TKA group [11].

While the above comparative clinical studies demonstrate equivalency with respect to functional outcomes, longer term survivorship in both institutional and national database registries demonstrate superiority in survivorship via smaller revision rates of CR TKAs compared to PS knees. Examining large institution registry data, Abdel et al examined the Mayo Clinic registry database and compared long-term outcomes of CR versus PS TKA designs. They evaluated 8117 TKAs and found that the 15-year survivorship was $90 \%$ for CR designs, compared to an inferior $77 \%$ for PS designs [12]. This intuitively makes sense that when there is a less mechanically dependent design that there will be less long-term failure of these components. The authors further reported that when accounting for age, sex, diagnosis and deformity, the risk for revision is substantially lower with CR designs [12]. These finding are observed and supported in larger national joint replacement registries as well. The Australian Registry data shows that out to fourteen years clinical follow up, the revision rate of CR knees is substantially less than PS knees (Figure 2). When accounting for patellar resurfacing, the lowest 
rate of revision is seen in CR knees where the patella is resurfaced (Figure 3). This data was formally reported by Vertullo et al, who documented in over 63,000 TKAs a $45 \%$ higher risk of revision for the patients with a PS knee design compared to a CR design [13].

\section{Conclusions}

Emerging clinical data over the past decade has shown equivalence of CR and CS designs as compared to PS designed articulations, while longer term registry studies report superiority of survivorship in TKAs with a CR bearing. Several potential complications of the cam and post mechanism have been reported in multiple designs. The downside of the increased mechanical articulation in a PS design may become even more apparent in the future with new technologies such as cementless fixation and highly-crosslinked polyethylene, which may prove less reliable in a suboptimal environment of increased mechanical stresses. TKA in younger patients may also be a reason to avoid PS designs, as the wear and fatigue would be propagated over a longer lifetime of the implant. As always, surgical technique is critical to long term success of these implants and appropriate balance in both flexion and extension is mandatory for optimal longevity and patient outcomes. Equivalent functional outcomes in multiple studies comparing CR-type bearings and PS TKA designs, combined with the potential deleterious outcomes associated with the cam-post articulation of PS designs and increased risk of revision and decreased long-term survivorship, preclude the need for PS TKA designs in the modern healthcare environment. Therefore, based on the available data and modern metrics, it can be concluded that traditional PS TKA designs with a cam and post articulation are truly "vestigial organs", and should be relegated to historical interest only in routine primary TKA. 


\section{Figures}

\section{Figure 1}

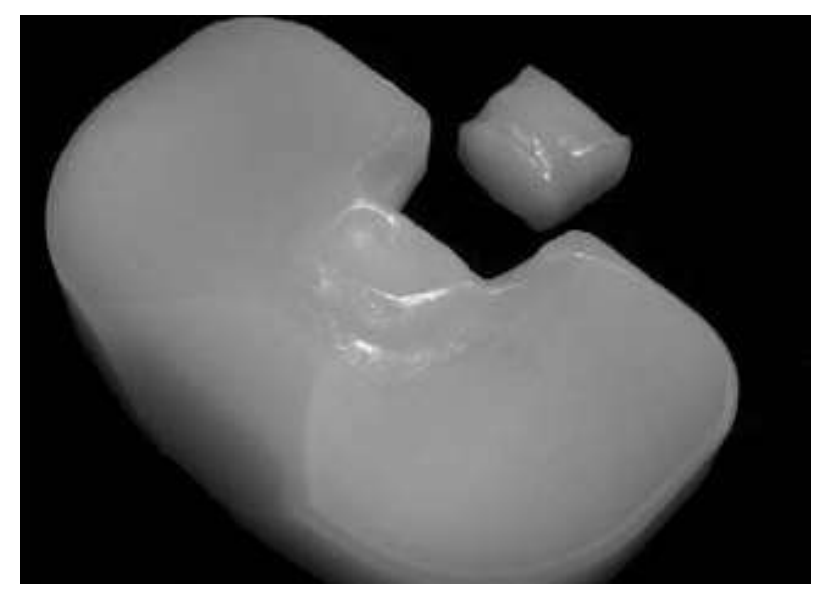

PS Polyethylene tray with broken post mechanism

\section{Figure 2}

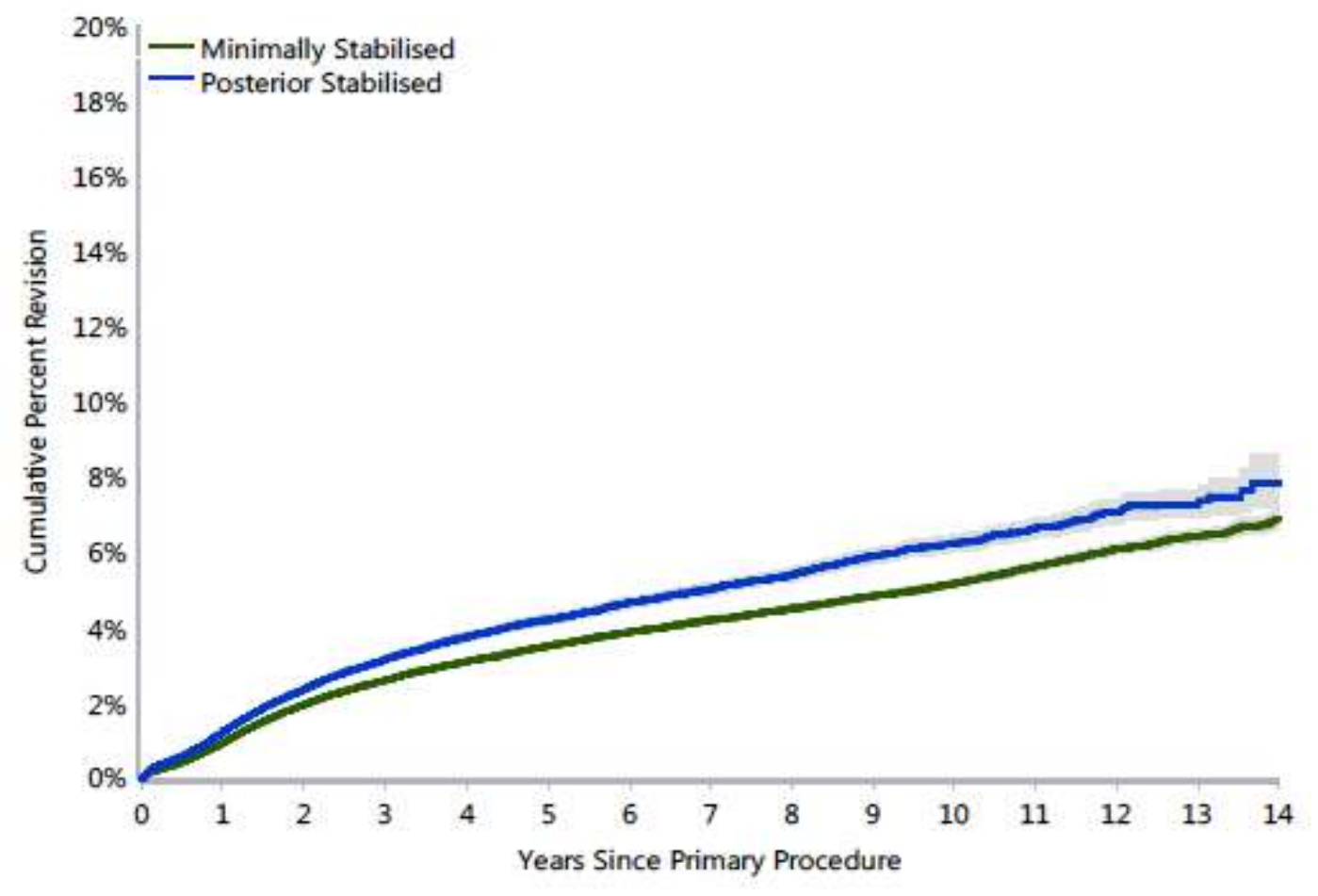

Australian Registry Data showing PS versus CR design cumulative percent revision 
Figure 3

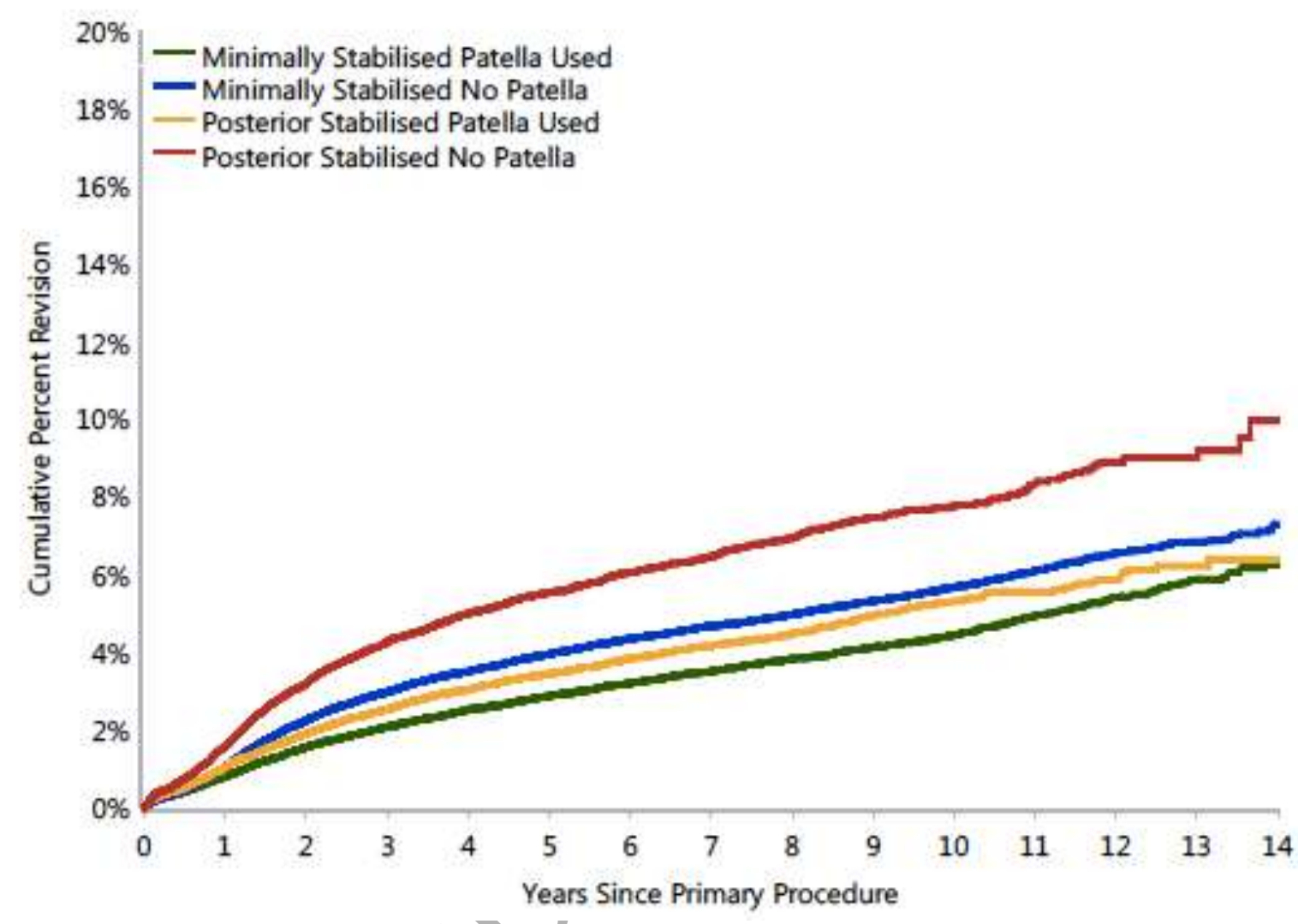

Australian Registry Data showing PS and CR design need for revision when factoring in patellar resurfacing. 


\section{References}

1. Alden, K.J., et al., Intraoperative fracture during primary total knee arthroplasty. Clin Orthop Relat Res, 2010. 468(1): p. 90-5.

2. Scott, D.F. and R.R. Smith, A prospective, randomized comparison of posterior stabilized versus cruciate-substituting total knee arthroplasty: a preliminary report with minimum 2-year results. J Arthroplasty, 2014. 29(9 Suppl): p. 179-81.

3. Lee, C.S., et al., Early nontraumatic fracture of the polyethylene tibial post in a NexGen LPS-Flex posterior stabilized knee prosthesis. J Arthroplasty, 2009. 24(8): p. 1292 e5-9.

4. Lim, H.C., et al., Fracture of a polyethylene tibial post in a Scorpio posterior-stabilized knee prosthesis. Clin Orthop Surg, 2009. 1(2): p. 118-21.

5. Lee, S.C., et al., Anterior dislocation after a posterior stabilized total knee arthroplasty. J Arthroplasty, 2012. 27(2): p. 324 e17-20.

6. Frye, B.M., et al., Effect of femoral component design on patellofemoral crepitance and patella clunk syndrome after posterior-stabilized total knee arthroplasty. J Arthroplasty, 2012. 27(6): p. 1166-70.

7. Biyani, R.K., et al., Does an Anterior-Lipped Tibial Insert Adequately Substitute for a Post-Cam Articulation in Total Knee Arthroplasty. Surg Technol Int, 2017. 30: p. 341345.

8. Parsley, B.S., et al., Posterior cruciate ligament substitution is not essential for excellent postoperative outcomes in total knee arthroplasty. J Arthroplasty, 2006. 21(6 Suppl 2): p. 127-31.

9. $\quad$ Laskin, R.S., et al., Deep-dish congruent tibial component use in total knee arthroplasty: a randomized prospective study. Clin Orthop Relat Res, 2000(380): p. 36-44.

10. Sur, Y.J., et al., Condylar-stabilizing tibial inserts do not restore anteroposterior stability after total knee arthroplasty. J Arthroplasty, 2015. 30(4): p. 587-91.

11. Scott, D.F., Prospective Randomized Comparison of Posterior-Stabilized Versus Condylar-Stabilized Total Knee Arthroplasty: Final Report of a Five-Year Study. J Arthroplasty, 2018. 33(5):p. 1384-1388.

12. Abdel, M.P., et al., Increased long-term survival of posterior cruciate-retaining versus posterior cruciate-stabilizing total knee replacements. J Bone Joint Surg Am, 2011. 93(22): p. 2072-8.

13. Vertullo, C,J., et al., The Effect on Long-Term Survivorship of Surgeon Preference for Posterior-Stabilized or Minimally Stabilized Total Knee Replacement: An Analysis of 63,416 Prostheses from the Australian Orthopaedic Association National Joint Replacement Registry. J Bone Joint Surg Am, 2017. 99(13): p. 1129-1139. 